\title{
Relación entre Perfil de Ingreso y Rendimiento Académico Línea Curricular Morfofunción. Carrera de Odontología, Universidad de La Frontera-Chile
}

\author{
Relationship Between Applicant's Profile and Academic Performance, Curricular \\ Design Morphofunction, School of Dentistry, Universidad de La Frontera-Chile
}

Josefina del Pilar Contreras Arellano; Héctor Paulo Sandoval Vidal \& Luis Alberto González Osorio

CONTRERAS, A. J. P.; SANDOVAL, V. H. P. \& GONZÁLEZ, O. L. A. Relación entre perfil deingreso y rendimiento académico línea curricular morfofunción. Carrera de Odontología, Universidad de La Frontera. Int. J. Odontostomat., 14(3):417423, 2020.

RESUMEN: El Rendimiento Académico es un concepto multifactorial en el que inciden aspectos internos, como las características propias del estudiante, y externos como el tipo de diseño curricular de cada institución. El propósito de este trabajo fue mejorar la disposición de recursos de apoyo educativo en estudiantes en riesgo de reprobación, con el objeto de elevar la calidad de la formación profesional de los odontólogos de la Universidad de La Frontera, identificando aquellos factores del Perfil de Ingreso que influyen en el Rendimiento Académico durante los primeros tres años de la carrera. Estudio cuantitativo observacional analítico con diseño de Cohorte Retrospectiva, la población fueron 222 estudiantes de odontología de las cohortes 2014, 2015 y 2016. Las variables estudiadas fueron promedio de PSU, promedio NEM, promedio Ranking, género, ascendencia mapuche, región de procedencia, establecimiento de procedencia (según tipo de establecimiento y régimen educativo) y quintil de ingreso. Se obtuvo que el promedio PSU solo influye en el primer semestre de la carrera $(p=0,000)$; el promedio NEM presentó una correlación positiva en el quinto $(p=0,002)$ y sexto semestre $(p=0,048)$; el promedio Ranking mostró una correlación positiva en el quinto semestre $(p=0,005)$; las diferencias por género fueron estadísticamente significativas del tercer semestre al sexto $(p=0,012 ; p=0,041 ; p=0,000 ; p=0,006$ respectivamente); según tipo de establecimiento las diferencias son estadísticamente significativas en los dos primeros semestres $(p=0,009 ; p=0,020)$ y en el cuarto $(p=0,038)$ y quinto semestre $(p=0,011)$; existe diferencia estadísticamente significativa entre los establecimientos Particulares Subvencionado y los Municipales en el Área de Ciencias Básicas $(p=0,010)$; la región de procedencia, el quintil de ingreso y la ascendencia mapuche no tiene incidencia en el RA. Valor de $p<0,05$ umbral para la significancia estadística.

PALABRAS CLAVE: rendimiento académico, educación, estudiantes, escuela de odontología.

\section{INTRODUCCIÓN}

El Rendimiento Académico (RA) es de naturaleza multidimensional lo que dificulta su delimitación tanto operacional como conceptual. González Barbera et al. (2012) propusieron dos formas de definir este concepto:

- Nivel de conocimientos y capacidades escolares exhibidas por estudiantes mediante distintos instrumentos de evaluación.

- Resultado global del estudiante que obtiene por medio de una valoración numérica comúnmente asignada por el docente, la cual se asocia a un proceso de instrucción específica.
Esta última definición es una conceptualización del RA a partir de su evaluación, pero es importante considerar que, al calificarlo, no solo influye el desempeño individual del estudiante sino también la manera en cómo es afectado por sus características, por el grupo de pares, el aula o el propio contexto educativo (Erazo-Santander, 2011).

Por otro lado, hay autores que indican que el RA es la expresión de habilidades, actitudes y valores que el estudiante va desarrollando a través del proceso de enseñanza-aprendizaje, de las acciones dirigidas a la explicación e interpretación de lo aprendido y la simplificación de éstos en valores cuantitativos y cualitativos.

Facultad de Odontología, Universidad de La Frontera, Temuco, Chile. 
CONTRERAS, A. J. P.; SANDOVAL, V. H. P. \& GONZÁLEZ, O. L. A. Relación entre perfil deingreso y rendimiento académico línea curricular morfofunción. Carrera de Odontología, Universidad de La Frontera. Int. J. Odontostomat., 14(3):417-423, 2020.

Los factores que influyen en el RA se pueden categorizar en 5 grandes grupos: variables de identificación, psicológicas, académicas, pedagógicas y socio familiares (Avendaño et al., 2016).

El estudio de predictores del RA es relevante en educación médica, ya que al ser el rendimiento un concepto multifactorial, no solo puede ser analizado desde las calificaciones, sino que debe considerar otras variables como el contexto de aprendizaje, la experiencia previa del estudiante y las estrategias cognitivas de cada uno (Zúñiga et al., 2009).

Para definir el Perfil de Ingreso de los estudiantes que entran a la carrera de Odontología de la Universidad de La Frontera (UFRO), en este trabajo se consideraron antecedentes sociodemográficos y rendimiento previo.

Dentro de los antecedentes sociodemográfico se incluyeron: las características del establecimiento de procedencia según: tipo de establecimiento (humanista-científico y técnico profesional), el régimen educativo del establecimiento (municipalizado, particular subvencionado y particular pagado); el género, la ascendencia mapuche declarada y la procedencia. $Y$ dentro de las condiciones socioeconómicas del estudiante, se consideró el quintil de ingreso o capacidad económica del hogar.

Por otro lado, el rendimiento previo o condiciones académicas de ingreso consideró el puntaje obtenido en la Prueba de Selección Universitaria (PSU), el promedio de Notas de Enseñanza Media (NEM) y el promedio Ranking.

En los primeros 6 semestres de la carrera de Odontología en la UFRO se dictan las asignaturas con mayor variabilidad en las calificaciones de los estudiantes y todas ellas corresponden a la Línea Curricular de Morfofunción (Tabla I). Esta línea curricular reúne disciplinas de las ciencias básicas y ciencias biomédicas, con el objetivo de lograr una formación científica que permita comprender y solucionar problemas del área discipli- nar y otorga la base conceptual necesaria para la formación de un profesional del área de la salud, en los aspectos anatómicos, fisiológicos y fisiopatológicos de los tejidos y de los diferentes sistemas que constituyen el cuerpo humano, estudiados a nivel macro y micro, contemplando bases microbiológicas y farmacología.

Para relacionar asignaturas de distintos semestres con las variables expuestas, se seleccionaron algunas, se agruparon en tres áreas: Ciencias Básicas (Química, Física, Química Orgánica y Bioquímica), Anatomía (Anatomía General, Anatomía Topográfica, Embriología, Histología, Neuroanatomía y Anatomía Patológica) y Fisiología (Fisiología Bucal, Fisiopatología y Patología General).

El objetivo de este estudio fue determinar la relación entre el Perfil de Ingreso de estudiantes de la Carrera de Odontología de la Universidad de La Frontera y el Rendimiento Académico en las asignaturas de la línea curricular de Morfofunción en tres cohortes.

\section{MATERIAL Y MÉTODO}

Se llevó a cabo un estudio de carácter cuantitativo, diseño observacional analítico en el cual se recopiló información de 222 estudiantes admitidos en la carrera de Odontología de la Universidad de La Frontera los años 2014 (86 individuos), 2015 (67 individuos) y 2016 (69 individuos).

La información se obtuvo a través de una base de datos anónima entregada por la Vicerrectoría de Pregrado de la Universidad de La Frontera, Chile.

Se consideró como indicador de RA el promedio ponderado semestral, el cual se calculó con las notas finales de las asignaturas de la línea curricular de Morfofunción por semestre. Esta variable se cuantificó numéricamente de 1 a 7.

Tabla I. Asignaturas de la Línea Curricular de Morfofunción de la Carrera de Odontología de la Universidad de La Frontera, Chile distribuidas de acuerdo al semestre en que se dictan.

\begin{tabular}{llllll}
\hline \multicolumn{1}{c}{$1^{\circ}$ Semestre } & $2^{\circ}$ Semestre & $3^{\circ}$ Semestre & $4^{\circ}$ Semestre & $5^{\circ}$ Semestre & $6^{\circ}$ Semestre \\
\hline Anatomía General & Anatomía & Embriología, & Neuroanatomía & Patología General & Anatomía \\
& $\begin{array}{l}\text { Topográfica de } \\
\text { Cabeza y Cuello }\end{array}$ & $\begin{array}{l}\text { Crecimiento y } \\
\text { Desarrollo }\end{array}$ & & & Patológica \\
Biología & Histología & Fisiología & Fisiopatología & Microbiología & Farmacología \\
$\begin{array}{l}\text { Física Aplicada } \\
\text { Química General }\end{array}$ & Química Orgánica & Bioquímica & Fisiología Bucal & & \\
\end{tabular}

Fuente: Elaboración propia.

Fuente: https://derribandomitos.ufro.cl/odontologia 
La clasificación de las variables que se compararon con el RA y se consideraron parte del Perfil de Ingreso de los estudiantes en estudio se presenta en la Fiqura 1.

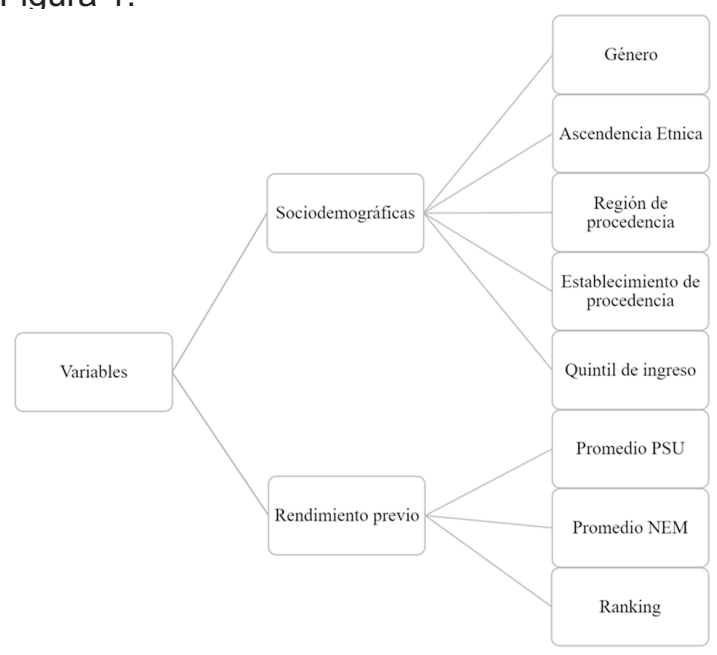

Fig. 1. Clasificación de las variables que delimitan el Perfil de Ingreso a la carrera de Odontología de la Universidad de La Frontera, Chile.

Análisis Estadístico: La recolección de los datos se registró en una planilla Microsoft Office Excel, se realizó un análisis descriptivo de los datos para los que se determinó el promedio y su respectiva desviación estándar, se realizó la prueba paramétrica t test para muestras independientes, ANOVA de un factor, la prueba de post hoc de Bonferroni y correlaciones bivariadas entre variables. Para el análisis de los datos se usó el

Tabla II. Correlación entre el Promedio PSU y el RA.

\begin{tabular}{|c|c|c|c|c|c|c|c|c|}
\hline & & PROM_PSU & RA1 & RA2 & RA3 & RA4 & RA5 & RA6 \\
\hline \multirow[t]{3}{*}{ PROM_PSU } & Correlación de Pearson & 1 & $0,251^{* *}$ & $-0,004$ & $-0,010$ & $-0,055$ & $-0,054$ & $-0,046$ \\
\hline & Sig. (bilateral) & 222 & 0,000 & 0,957 & 0,887 & 0,454 & 0,474 & 0,585 \\
\hline & $\mathrm{N}$ & & 222 & 204 & 194 & 189 & 178 & 143 \\
\hline
\end{tabular}

**La correlación es significativa en el nivel 0,01 (bilateral).

Tabla III. Correlación entre el Promedio NEM y el RA.

\begin{tabular}{llcllllll}
\hline & & PROM_NEM & RA1 & RA2 & RA3 & RA4 & RA5 & RA6 \\
\hline PROM_NEM & Correlación de Pearson & 1 & $-0,041$ & 0,091 & 0,118 & 0,114 & $0,234^{* *}$ & $0,166^{*}$ \\
& Sig. (bilateral) & & 0,540 & 0,193 & 0,101 & 0,118 & 0,002 & 0,048 \\
& N & 222 & 222 & 204 & 194 & 189 & 178 & 143 \\
\hline
\end{tabular}

*La correlación es significativa en el nivel 0,05 (bilateral). **La correlación es significativa en el nivel 0,01 (bilateral).

Tabla IV. Correlación entre el Promedio Ranking y el RA.

\begin{tabular}{lllllllll}
\hline & & $\begin{array}{c}\text { PROM } \\
\text { Ranki }\end{array}$ & RA1 & RA2 & RA3 & RA4 & RA5 & RA6 \\
& & $-{ }^{2}$ & & & & \\
\hline PROM_Rankin & Correlación de & 1 & $-0,153^{*}$ & 0,076 & 0,110 & 0,108 & $0,209^{* *}$ & 0,149 \\
& Sig. (bilateral) & & 0,023 & 0,278 & 0,126 & 0,140 & 0,005 & 0,076 \\
& N & 222 & 222 & 204 & 194 & 189 & 178 & 143 \\
\hline
\end{tabular}

*La correlación es significativa en el nivel 0,05 (bilateral). ** La correlación es significativa en el nivel 0,01 (bilateral). 
Relación entre el género y el RA: la distribución por género es de 95 hombres y 127 mujeres. El promedio de notas correspondientes a la línea de Morfofunción en cada semestre tiene un rango de 4,4 a 4,9 (Tabla V).

Tabla V. Promedio de notas por semestre según Género.

\begin{tabular}{llrccc}
\hline \multicolumn{2}{c}{ Género } & N & Media & $\begin{array}{c}\text { Desviación } \\
\text { Estándar }\end{array}$ & $\begin{array}{c}\text { Media de error } \\
\text { estándar }\end{array}$ \\
\hline RA1 & Hombre & 95 & 4,4 & 0,63 & 0,06 \\
& Mujer & 127 & 4,4 & 0,47 & 0,04 \\
RA2 & Hombre & 84 & 4,6 & 0,49 & 0,05 \\
& Mujer & 120 & 4,7 & 0,48 & 0,04 \\
RA3 & Hombre & 77 & 4,5 & 0,31 & 0,04 \\
& Mujer & 117 & 4,7 & 0,45 & 0,04 \\
RA4 & Hombre & 73 & 4,7 & 0,38 & 0,04 \\
& Mujer & 116 & 4,8 & 0,54 & 0,05 \\
RA5 & Hombre & 68 & 4,6 & 0,54 & 0,07 \\
& Mujer & 110 & 4,9 & 0,51 & 0,05 \\
RA6 & Hombre & 51 & 4,4 & 0,38 & 0,05 \\
& Mujer & 92 & 4,6 & 0,45 & 0,05 \\
\hline
\end{tabular}

De acuerdo a la prueba $t$ de muestras independientes realizada las diferencias son estadísticamente significativas a partir del tercer semestre (Tabla VI).

Relación entre Ascendencia Mapuche y RA: el $14,9 \%$ de los estudiantes declararon poseer Ascendencia Mapuche. El RA por semestre es similar entre los estudiantes con o sin Ascendencia Mapuche y el promedio de notas va de 4,3 a 4,9. Solo en el quinto semestre hay diferencia estadísticamente significativa en los promedios (Tabla VII), aquellos con Ascendencia Mapuche presen$\tan$ un 4,6 y los $\sin$, un 4,8 .

Relación entre el Tipo de Establecimiento de procedencia y el RA: del total de la muestra, 216 estudiantes provienen de establecimientos Científico Humanista y 6 , de Técnico Profesional. El promedio en cada semestre tiene un rango de 3,8 a 4,8 .

Tabla VI. Prueba t de muestras independientes para relación entre Género y RA.

\begin{tabular}{|c|c|c|c|c|c|c|}
\hline & \multicolumn{6}{|c|}{ prueba t para la igualdad de medias } \\
\hline & \multirow[t]{2}{*}{$\mathrm{t}$} & \multirow[t]{2}{*}{ gl } & \multirow[t]{2}{*}{ Sig. (bilateral) } & \multirow{2}{*}{$\begin{array}{l}\text { Diferencia de } \\
\text { medias }\end{array}$} & \multicolumn{2}{|c|}{$95 \%$ de intervalo de confianza } \\
\hline & & & & & Inferior & Superior \\
\hline RA1* $^{*}$ & $-0,008$ & 167,6 & 0,994 & $-0,0006$ & $-0,15$ & 0,15 \\
\hline RA2** & $-0,877$ & 202 & 0,381 & $-0,0603$ & $-0,20$ & 0,08 \\
\hline RA3* $^{*}$ & $-2,54$ & 191,4 & 0,012 & $-0,1378$ & $-0,24$ & $-0,03$ \\
\hline $\mathbf{R A 4}^{* *}$ & $-2,06$ & 187 & 0,041 & $-0,1484$ & $-0,29$ & $-0,01$ \\
\hline $\mathbf{R A 5}^{* *}$ & $-4,24$ & 176 & 0,000 & $-0,3386$ & $-0,50$ & $-0,18$ \\
\hline $\mathbf{R A 6}^{* *}$ & $-2,80$ & 141 & 0,006 & $-0,2082$ & $-0,36$ & $-0,06$ \\
\hline
\end{tabular}

*No se asumen varianzas homogéneas. ${ }^{*}$ Se asumen varianzas homogéneas.

Tabla VII. Prueba t de muestras independientes para la relación entre Ascendencia Mapuche y RA.

\begin{tabular}{|c|c|c|c|c|c|c|}
\hline & \multicolumn{6}{|c|}{ Prueba t para la igualdad de medias } \\
\hline & \multirow[t]{2}{*}{$\mathrm{t}$} & \multirow[t]{2}{*}{ gl } & \multirow[t]{2}{*}{ Sig. (bilateral) } & \multirow{2}{*}{$\begin{array}{l}\text { Diferencia de } \\
\text { medias }\end{array}$} & \multicolumn{2}{|c|}{$95 \%$ de intervalo de confianza } \\
\hline & & & & & Inferior & Superior \\
\hline $\mathrm{RA}^{*}{ }^{*}$ & 0,809 & 38,80 & 0,424 & 0,100 & $-0,15$ & 0,35 \\
\hline $\mathrm{RA} 2^{* *}$ & 0,994 & 202 & 0,322 & 0,0949 & $-0,09$ & 0,28 \\
\hline $\mathrm{RA}^{* *}$ & 1,31 & 192 & 0,191 & 0,1074 & $-0,05$ & 0,27 \\
\hline $\mathrm{RA}^{* *}$ & 1,87 & 187 & 0,063 & 0,1883 & $-0,01$ & 0,39 \\
\hline $\mathrm{RA}^{* *}$ & 2,33 & 176 & 0,021 & 0,2653 & 0,04 & 0,49 \\
\hline RA6** & 0,231 & 141 & 0,749 & 0,0363 & $-0,19$ & 0,26 \\
\hline
\end{tabular}

*No se asumen varianzas homogéneas. **Se asumen varianzas homogéneas.

Tabla VIII. Prueba t de muestras independientes para la relación entre Tipo de Establecimiento de Procedencia y RA. Se asumen varianzas homogéneas.

\begin{tabular}{|c|c|c|c|c|c|c|}
\hline & \multicolumn{6}{|c|}{ Prueba t para la igualdad de medias } \\
\hline & \multirow[t]{2}{*}{$\mathrm{t}$} & \multirow[t]{2}{*}{ gl } & \multirow[t]{2}{*}{ Sig. (bilateral) } & \multirow[t]{2}{*}{ Diferencia de } & \multicolumn{2}{|c|}{$95 \%$ de intervalo de confianza } \\
\hline & & & & & Inferior & Superior \\
\hline RA1 & 2,655 & 220 & 0,009 & 0,5889 & 0,15 & 1,03 \\
\hline RA2 & 2,345 & 202 & 0,020 & 0,4647 & 0,07 & 0,86 \\
\hline RA3 & 1,781 & 192 & 0,076 & 0,2950 & $-0,03$ & 0,62 \\
\hline RA4 & 2,090 & 187 & 0,038 & 0,4572 & 0,03 & 0,89 \\
\hline RA5 & 2,565 & 176 & 0,011 & 0,6217 & 0,14 & 1,10 \\
\hline RA6 & 0,324 & 141 & 0,746 & $-0,1011$ & $-0,52$ & 0,72 \\
\hline
\end{tabular}


De acuerdo a la prueba t de muestras independientes realizada las diferencias son estadísticamente significativas en los dos primeros semestres y en el cuarto y quinto semestre (Tabla VIII).

Relación entre Régimen Educativo y Áreas de Estudio: la distribución por Régimen Educativo corresponde a 153 estudiantes provenientes de establecimientos Particulares Subvencionados, 56 estudiantes de establecimientos Municipales y 11 de establecimientos Particulares Pagados.

En la Tabla IX se presenta el promedio de notas por Área de Estudio, siendo el mayor promedio en Fi- siología un 4,7, y el menor promedio es de Ciencias Básicas con un 4,3. Los rangos de distribución van desde el 1,5 al 6,1. En la Tabla $X$ se muestra el análisis ANOVA para muestras independientes encontrándose que hay diferencias significativas en Ciencias Básicas.

Con la aplicación de la prueba de Bonferroni (Tabla XI) se estableció que la diferencia antes nombrada es estadísticamente significativa entre los establecimientos Particulares Subvencionado y los Municipales, siendo los Particulares Subvencionados los con mejor promedio (Tabla IX).

Tabla IX. Promedio de notas por Área de estudio.

\begin{tabular}{lccccccccc}
\hline & \multicolumn{4}{c}{ Ciencias Básicas } & \multicolumn{3}{c}{ Anatomía } & \multicolumn{3}{c}{ Fisiología } \\
& Promedio & Mín & Máx & Promedio & Mín & Máx & Promedio & Mín & Máx \\
\hline Particular Subvencionado & 4,6 & 1,9 & 6,1 & 4,6 & 1,6 & 5,6 & 4,7 & 1,5 & 5,9 \\
Municipal & 4,3 & 2,7 & 5,3 & 4,5 & 3,5 & 5,5 & 4,7 & 3,4 & 5,6 \\
Particular Pagado & 4,4 & 2,2 & 6,0 & 4,4 & 2,9 & 6,0 & 4,6 & 4,3 & 5,0 \\
\hline
\end{tabular}

\begin{tabular}{|c|c|c|c|c|c|c|}
\hline & & $\begin{array}{l}\text { Suma de } \\
\text { cuadrados }\end{array}$ & gl & $\begin{array}{c}\text { Media } \\
\text { cuadrática }\end{array}$ & $\mathrm{F}$ & Sig. \\
\hline Ciencias & Entre grupos & 3,223 & 2 & 1,612 & 4,706 & 0 , \\
\hline \multirow[t]{2}{*}{ Básicas } & Dentro de grupos & 73,625 & 2 & 0,342 & & \\
\hline & Total & 76,848 & 2 & 0,137 & & \\
\hline \multirow[t]{3}{*}{ Anatomía } & Entre grupos & 0,273 & 2 & 0,247 & 0,553 & 0 \\
\hline & Dentro de grupos & 53,596 & 2 & & & \\
\hline & Total & 53,870 & 2 & & & \\
\hline \multirow[t]{3}{*}{ Fisiología } & Entre grupos & 0,377 & 2 & 0,189 & 0,852 & 0 \\
\hline & Dentro de grupos & 41,195 & 1 & 0,221 & & \\
\hline & Total & 41,572 & 8 & & & \\
\hline
\end{tabular}

Tabla XI. Prueba de Bonferroni para relación entre Áreas de Estudio y Régimen Educativo.

\begin{tabular}{|c|c|c|c|c|c|c|c|}
\hline \multirow[t]{2}{*}{$\begin{array}{l}\text { Variable } \\
\text { dependiente }\end{array}$} & \multirow[t]{2}{*}{ (I) Reg_Educativo } & \multirow[t]{2}{*}{ (J)Reg_Educativo } & \multirow[t]{2}{*}{$\begin{array}{l}\text { Diferencia de } \\
\text { medias (I-J) }\end{array}$} & \multirow[t]{2}{*}{$\begin{array}{l}\text { Error } \\
\text { estándar }\end{array}$} & \multirow[t]{2}{*}{ Sig. } & \multicolumn{2}{|c|}{$\begin{array}{c}\text { Intervalo de } \\
\text { confianza al } 95 \%\end{array}$} \\
\hline & & & & & & $\begin{array}{l}\text { Límite } \\
\text { inferior }\end{array}$ & $\begin{array}{c}\text { Límite } \\
\text { superior }\end{array}$ \\
\hline C.BÁSICAS & $\begin{array}{l}\text { Particular } \\
\text { Subvencionado }\end{array}$ & Municipal & $0,27356^{*}$ & 0,09156 & 0,009 & 0,0526 & 0,4945 \\
\hline & Municipal & Particular Pagado & $-0,07338$ & 0,19299 & 1,000 & $-0,539$ & 0,3923 \\
\hline & Particular Pagado & $\begin{array}{l}\text { Particular } \\
\text { Subvencionado }\end{array}$ & $-0,20018$ & 0,18275 & 0,824 & $-0,641$ & 0,2408 \\
\hline
\end{tabular}

La diferencia de medias es significativa en el nivel $0,05^{\star}$.

\section{DISCUSIÓN}

De acuerdo a los resultados obtenidos y en concordancia con lo propuesto por diversos autores (Rocha et al., 2009; Medina \& Flores, 2012) existe una baja correlación positiva, pero estadísticamente significativa entre el RA en las asignaturas correspondientes a la línea de Morfofunción y el Promedio PSU en 
CONTRERAS, A. J. P.; SANDOVAL, V. H. P. \& GONZÁLEZ, O. L. A. Relación entre perfil deingreso y rendimiento académico línea curricular morfofunción. Carrera de Odontología, Universidad de La Frontera. Int. J. Odontostomat., 14(3):417-423, 2020.

el primer semestre de la carrera, lo que difiere de lo escrito por Barahona \& Aliaga (2013), quienes no encontraron relación.

El Promedio Ranking al ser un puntaje relacionado con la posición del estudiante dentro de su establecimiento educacional comparándolo con generaciones previas del mismo (Rodríguez Garcés \& Padilla Fuentes, 2016; Faúndez et al., 2017), dificulta la comparación entre individuos de diferentes colegios. El análisis de este trabajo entregó que el Promedio Ranking tiene correlación negativa con el RA en el primer semestre y positiva en el quinto semestre.

Estudios afirman que el Promedio NEM es un buen predictor del RA en los primeros años de estudio universitario (Rocha et al., 2009; Medina \& Flores; Soria-Barreto \& Zúñiga-Jara, 2014; Rowland \& Rieken, 2018), sin embargo, en este análisis se obtuvo lo contrario, que coincide con lo descrito por Medina Moreno et al. (2014) ya que, solo en el tercer año se encontró baja correlación positiva.

La razón por la que el promedio NEM se relacionaría con un buen rendimiento en los primeros años universitarios puede deberse a los estilos de aprendizaje, a los hábitos de estudio u otros aspectos, los cuales se replicarían al ingresar a la Universidad, sin embargo, estas variables no fueron incorporados en el estudio porque no se encuentran disponible en la base de datos utilizada.

En relación a la comparación del RA según género, a partir del tercer semestre hay diferencias estadísticamente significativas entre hombres y mujeres, siendo las mujeres quienes presentan mejores promedios. Esto coincide con lo encontrado en el estudio Pérez-Cárceles et al. (2014).

Debido al alto porcentaje de población Mapuche en Temuco, se consideró relevante analizar la relación entre la ascendencia de esta etnia y el RA en los estudiantes que la declararan, y se obtuvo que solo en el quinto semestre de la carrera hay diferencia estadísticamente significativa, siendo aquellos estudiantes con ascendencia declarada los que obtuvieron RA más bajo. Al ser solo un semestre en el que se encontraron diferencias significativas, se puede concluir que a pesar de las debilidades académicas de ingreso que presentan los estudiantes con Ascendencia Mapuche (Navarrete et al., 2013), el RA en las asignaturas de Morfofunción es similar entre quienes la declaran y los que no.
Aquellos estudiantes provenientes de Establecimientos Científicos Humanistas obtienen mejor RA que aquellos de colegios Técnico Profesional ya que, en los primeros se entregan contenidos diversos para que el estudiante pueda seguir sus estudios en alguna Universidad, en cambio los establecimientos Técnicos Profesionales constituyen un ámbito de preparación inicial para una vida de trabajo y se construye articulando el dominio de las competencias propias de una especialidad, con el aprendizaje tanto de los objetivos transversales como de los objetivos y contenidos de la Formación General de la Educación Media (Ministerio de Educación, 2013). Según el Régimen de Estudio del establecimiento, aquellos estudiantes procedentes de colegios Particulares Subvencionados, obtuvieron mejor RA en las asignaturas de Morfofunción en comparación con los de colegios Municipales y Particulares Pagados.

Al agrupar asignaturas por áreas de estudio, la categoría Ciencias Básicas incluyó Física y Química General las cuales presentan alto porcentaje de reprobación en las cohortes estudiadas (Tabla II) y al analizarlas junto al Régimen Educativo se obtuvo que es el área con RA más bajo. Por lo tanto, estas asignaturas pueden ser objeto de estudio por parte de las autoridades universitarias para incluir estrategias que lleven a mejorar el RA de los estudiantes y disminuir el porcentaje de reprobación, como por ejemplo talleres de nivelación.

En relación al Nivel Socioeconómico (analizado según Quintil de Ingreso) y la Región de Procedencia, al contrario de lo que se pensaba previo al estudio, no existe correlación entre estos aspectos y el RA de los estudiantes de Odontología, esto difiere con lo expuesto por Gonzáles (2015), quien en su estudio encontró que el nivel socioeconómico bajo presenta correlación con un bajo RA en universitarios.

Las limitaciones de este estudio son las propias del diseño de Cohorte Retrospectiva, ya que al estudiar aspectos pasados de una población específica se dificulta el control de la pérdida de datos. Por otro lado, la base de datos consultada es acotada a lo que los estudiantes responden en la encuesta previa a la entrada a la UFRO por lo que hay aspectos como los hábitos de estudio, los estilos de aprendizajes, contención familiar y actividad laboral que no se incluyeron en este estudio y podrían ser interesantes de analizar en otra investigación.

AGRADECIMIENTOS. Al Profesor Pablo Navarro Cáceres por su invaluable ayuda en el análisis clínico. 
CONTRERAS, A. J. P.; SANDOVAL, V. H. P. \& GONZÁLEZ, O. L. A. Relationships between applicant's profile and academic performance, in curricular design morphofunction, School of Dentistry, Universidad de La Frontera. Int. J. Odontostomat., 14(3):417-423, 2020.

ABSTRACT: Academic Performance is a multifactorial concept in which internal factors, such as the student's personal characteristics, and external elements, such as the curricular design of each educational institution, come into play. The purpose of this essay was to improve the resources of educational support for students at risk of failure, in order to strengthen the quality of the professional training of dentists at Universidad de la Frontera, identifying key factors in Applicant's Profiles that affect Academic Performance during the first three years of studies. Analytic quantitative observational study with Retrospective Cohort design, the population consisted of 222 Dentistry students of 2014,2015 , and 2016 cohorts. The analyzed variables were the scholastic aptitude test (PSU) average, NEM average, class ranking average, sex, Mapuche ethnicity, region of provenance, establishment of origin (according to type of establishment and educational regime) and income quintile. Results showed that PSU scores only affect the first semester of studies $(p=0,000)$; NEM score presented a positive correlation in the fifth $(p=0,002)$ and sixth semester $(p=0,048)$; class ranking showed a positive correlation in fifth semester $(p=0,005)$; sex differences were statistically significant from the third until the sixth semester $(p=0,012 ; p=0,041 ; p=0,000$; $p=0,006$ respectively); differences in the type of establishments are statistically significant in the first two semesters $(p=0,009 ; p=0,020)$ and in the fourth $(p=0,038)$ and fifth semesters $(p=0,011)$; there exists statistically significant differences between Private Subsidized schools, and municipal schools in the area of Basic Sciences $(p=0,010)$; the region of provenance, the income quintile and Mapuche ethnicity do not impact the RA. Ap $<0,05$ threshold for statistical significance.

KEY WORDS: academic performance, education, students, school dentistry.

\section{REFERENCIAS BILIOGRÁFICAS}

Avendaño, C. A.; Gutiérrez, K. A.; Salgado, C. F. \& Alonso-DosSantos, M. Rendimiento académico en estudiantes de ingeniería comercial: modelo por competencias y factores de influencia. Form. Univ., 9(3):3-10, 2016

Barahona, U. P. \& Aliaga, V. Variables predictoras del rendimiento académico de los alumnos de primer año de las carreras de Humanidades de la Universidad de Atacama, Chile. Rev. Int. Investig. Cienc. Soc., 9(2): 207-20, 2013

Erazo-Santander, O. El rendimiento académico, un fenómeno de múltiples relaciones y complejidades. Rev. Vanguard. Psicol., 2(2):144-73, 2011.

Faúndez, G. R.; Labarca, T. J.; Cornejo, M. M. F.; Villarroel, J. M. \& Gil, L. F. J. Ranking 850, transición a la educación terciaria de estudiantes con desempeño educativo superior y puntaje PSU insuficiente. Pensam. Educ. Rev. Investig. Educ. Latinoam., 54(1):1-11, 2017.

González Barbera, C.; Caso Niebla, J.; Díaz López, K. \& López Ortega, M. Rendimiento académico y factores asociados. Aportaciones de algunas evaluaciones a gran escala. Bordon Rev. Pedag., 64(2):51-68, 2012.

Medina Moreno, A. P.; Aguirre Sanhueza, M. \& Luengo Machuca, L. Predictibilidad de las notas de enseñanza media según establecimiento de origen sobre el rendimiento académico en estudiantes de Odontología. Rev. Educ. Med. Super., 28(1):65-73, 2014.

Medina, A. \& Flores, F. ¿Predicen los requisitos de ingreso a odontología el rendimiento académico durante primer año?. J. Oral Res., 1(1):15-8, 2012.

Ministerio de Educación. Bases Curriculares para Formación Diferenciada Técnico - Profesional. Decreto Supremo de Educación N452/2013. Santiago de Chile, Ministerio de Educación, Gobierno de Chile, 2013.

Navarrete, S.; Candia, R. \& Puchi, R. Factores asociados a la deserción/retención de los estudiantes mapuche de la Universidad de la Frontera e incidencia de los programas de apoyo académico. Calid. Educ., (39):43-80, 2013.

Pérez-Cárceles. M. C.; Gómez-Gallego, M.; Gómez-Gallego, J. C.; Palazón-Pérez de los Cobos, A. \& Gómez-García, J. El género como variable moderadora de los resultados académicos en la enseñanza universitaria. EERS Reg. Sect. Econ. Stud., 14(3):5564, 2014.

Rocha, P. F.; Acevedo, P. C. G. y Flores, O. M. Perfi I de ingreso de los estudiantes de la Carrera de Tecnología Médica de la Universidad de Concepción (cohortes 2006 al 2008). Determinación de variables predictivas del rendimiento académico en asignaturas de ciencias. Rev. Educ. Cienc. Salud, 6(1):28-33, 2009.

Rodríguez Garcés, C. R. \& Padilla Fuentes, G. Trayectoria escolar y selección universitaria: comportamiento del ranking como factor de inclusión a la educación superior. Sophia, 12(2):195-206, 2016.

Rowland, K. C. \& Rieken, S. Rethinking dental school admission criteria: correlation between pre-admission variables and firstyear performance for six classes at one dental school. J. Dent. Educ., 82(4):411-6, 2018.

Soria-Barreto, K. \& Zúñiga-Jara, S. Aspectos determinantes del éxito académico de estudiantes universitarios. Form. Univ., 7(5):4150, 2014.

Zúñiga, D.; Mena, B.; Oliva, R.; Pedrals, N.; Padilla, O. \& Bitran, M. Modelos de predicción del rendimiento académico de los estudiantes de medicina en el ciclo básico y preclínico. Un estudio longitudinal. Rev. Med. Chile, 137(10):1291-300, 2009.

Dirección para correspondencia:

Mg. Paulo Sandoval Vidal

Facultad de Odontología

Universidad de La Frontera

Temuco

CHILE

Email: paulo.sandoval@ufrontera.cl

Recibido: 25-02-2020

Aceptado: 20-04-2020 